\title{
Geometrical optics modelling of grazing incidence X-ray fluorescence of nanoscaled objects
}

\author{
Stanisław H. Nowak, ${ }^{\text {*a }}$ Falk Reinhardt, ${ }^{\mathrm{b}}$ Burkhard Beckhoff, ${ }^{\mathrm{b}}$ Jean-Claude Dousse ${ }^{\mathrm{a}}$ \\ and Jakub Szlachetko ${ }^{\text {cd }}$ \\ X-ray Standing Wave (XSW) is a well established formalism for modelling Grazing Incidence X-ray \\ Fluorescence (GIXRF) experiments. However, when probing nanostructured surfaces with complex \\ morphology the effects of the interaction of the XSW with structure elements need to be investigated. \\ This is not always easy and sometimes even not possible. In the present work a novel approach \\ employing Geometrical Optics (GO) calculations is proposed. The model is employed for simulations of \\ two different types of nano-particles distributed on a flat surface. It is shown that GO simulation yields \\ results with good agreement when compared to absolute measurements even when XSW deteriorates.
}

\section{Introduction}

Artificial structures on wafer surfaces are becoming more and more sophisticated. The increasing ability to produce such patterned wafer surfaces calls for more evolved process analysis methods to further match the high standard in quality and reproducibility of the products. The characterisation of both nanoscaled structures and nanoparticles deposited on semiconductor surfaces requires reliable measurement procedures, quantification schemes and related validation.

One of the methods having the potential to effectively contribute to the characterisation of nanostructures deposited on flat surfaces is Grazing Incidence X-ray Fluorescence (GIXRF) analysis. ${ }^{1}$ GIXRF is based on the Total Reflection X-ray Fluorescence (TXRF) geometry which offers a high sensitivity to the near-surface area and low levels of detection in the picogram to femtogram range. Additionally, by varying the angle of incidence, the penetration depth into the substrate can be changed, as well as the excitation conditions above the substrate.

A very elegant and widely used way of interpreting the GIXRF measurements of, for example, nanoparticles on surfaces involves calculations of the so-called X-ray Standing Wave (XSW) field resulting from the interference between the incident beam and the part of the beam that is subject to external totalreflection. The change of the incident angle modifies the XSW field, i.e., the radiation intensity exciting the particles, yielding changes in the X-ray fluorescence signal. However, the XSW

${ }^{a}$ Physics Department, University of Fribourg, Ch. du Musée 3, CH-1700 Fribourg, Switzerland. E-mail: stanislaw.nowak@unifr.ch; Fax: +41 26300 9747; Tel: +41 26 3009027

${ }^{b}$ Physikalisch-Technische Bundesanstalt (PTB), Abbestr. 2-12, D-10587 Berlin, Germany

'Paul Scherrer Institut, CH-5232 Villigen PSI, Switzerland

${ }^{d}$ Institute of Physics, Jan Kochanowski University, 25-406 Kielce, Poland based theory treats the particles as a small perturbation, which is only valid if absorption and interference effects caused by the particles can be neglected. ${ }^{2}$ This requires small particle dimensions and large distances between them. For wide or densely distributed structures the XSW approximation has to be assumed to fail. However, a complete monolayer of densely packed nanoparticles can be again expected to be describable by the conventional XSW calculations.

Alternatively, provided the wavelength of the exciting radiation is much smaller than the size of the structures, the concept of Geometrical Optics (GO) can be used. Here, a GO-based ray tracing approach taking into account reflection, refraction, and absorption effects is considered. Assuming the optical path differences between different rays to be shorter than a predefined coherence length, the interference of different rays at a given point can be taken into consideration by simply summing the amplitudes of the corresponding waves. In the geometrical optics approach the XSW method can be seen as a limiting case of very small and sparsely distributed particles where absorption and multiple reflections inside the particles are negligible.

In this work, numerical GO calculations are introduced and compared to experimental GIXRF profiles and XSW simulations. It is shown that GO calculations agree well with experimental data and give more realistic results when compared to XSW simulations.

\section{Principles of GIXRF}

The operational principle of the GIXRF technique consists of measuring the intensity evolution of an X-ray fluorescence line when varying the angle of the incident X-ray beam around the critical angle $\varphi_{\mathrm{c}}$ for external total reflection at a flat substrate. For incidence angles below $\varphi_{\mathrm{c}}$, the beam is reflected at the substrate to almost $100 \%$. Both the incident beam and the 
reflected beam excite on-surface structures or particles yielding a two-fold excitation intensity. Also, due to the coherence of the exciting beam, there is a strong angle-dependence for the nearsurface intensity. The latter can be explained either by an emerging X-ray standing wave field that forms due to the interference of the incident and reflected beams or by optical path differences between incident rays and already reflected rays. This angle-dependent near-surface intensity expands into the substrate as an evanescent wave. By changing the angle of incidence beyond $\varphi_{\mathrm{c}}$ the incident beam will start to penetrate the substrate directly and the setup becomes highly sensitive also for the elemental composition below the surface. In short, TXRF denotes surface sensitive measurements of the X-ray fluorescence intensity at a fixed angle below $\varphi_{c}$, while in GIXRF measurements the fluorescence intensity is measured as a function of the incidence angle which is varied around the critical angle.

Depending on the sample morphology three principal types of GIXRF angular profiles are distinguished: bulk-like, layer-like and particle-like., ${ }^{1,3}$ For the latter two the shape of the GIXRF spectrum significantly changes when decreasing the surface structure size down to the nanometer regime. Then the coherence of the incident beam, which is usually in the range of a few hundred nanometers, ${ }^{2}$ has to be taken into account.

For the bulk structure the GIXRF angular profile approximately follows the transmission coefficient of the surface. In the layer-like structure for angles above $\varphi_{\mathrm{c}}$, a number of reflections occur resulting in interference fringes in the detected fluorescence. For particle-like structures the angular profile results from the sum of the direct and singly reflected radiation. Thereby, the detected X-ray intensity is increased and exhibits an interference pattern below $\varphi_{\mathrm{c}}$ of the support material.

\section{Ray tracing method}

The transition of the X-ray fluorescence emission from a particle-like to a layer-like morphology is still not fully understood. In the literature such samples with an intermediate morphology are treated as layer-like structures with the roughness introduced as a small perturbation of the interface potential (Nevot-Croce model), ${ }^{4}$ as a stack of layers with reduced average densities, ${ }^{5}$ or as a composition of layers of different thicknesses. ${ }^{6}$ These crude models, however, cannot be used for surfaces characterized by large roughnesses and/or highly correlated height distributions, ${ }^{7}$ or for grainy structures for which the incident X-ray radiation passes through several successive grains. ${ }^{6}$ For those sample systems the well-established XSW approach will yield reliable results only in very specific cases, e.g., surface depositions with low- $Z$ elements of neglectable absorption.

For X-rays with an energy above $1 \mathrm{keV}$, the wavelength $(\lambda<$ $1.2 \mathrm{~nm}$ ) is usually smaller than the dimensions of the investigated structures. Thus, the incident X-ray beam can be treated in a purely geometric approach, i.e., the geometrical optics ray tracing method. In general, the latter describes very well the effects of reflection, refraction, transmission and absorption. In this work a 2-dimensional reverse ray tracing approach is used which tracks the full evolution of plane waves from their source to the point, where they are absorbed. For nanoscaled structures, where absorption in the detection channel has a negligibly small effect, the number of detected fluorescence photons is directly proportional to the number of absorptions taking place in the sample. Therefore, in order to get the final radiation intensity, one has to consider all the possible ray paths between the source and a given absorption point, taking into account the interferences of the different rays. This is, however, a very complex and hardly solvable problem.

To significantly reduce the free parameters, we make two assumptions: (1) the exciting beam is perfectly parallel; (2) the on-surface objects have only horizontal and vertical interfaces. With these assumptions, for any given incident radiation direction, the refraction into the structure can be realized only on a horizontal or a vertical interface. Of course, the direction of a ray passing through the vacuum between two vertical interfaces of neighboring structure elements does not change.

In a 2-dimensional coordinate system, where the axis $x$ lies in the plane of the substrate surface and the axis $y$ is perpendicular to that interface, reflections at the horizontal interfaces change only the $y$-component of the wave vector, the length of the latter remains unchanged.

Reflections at vertical interfaces can be neglected as in the Xray regime the reflectivity quickly goes to zero for large angles of incidence. Thus, provided that a structure has a constant height, only four final ray path directions have to be considered: two ray path directions (up and down) for incident radiation reaching the structure from horizontal interfaces and two ray path directions (up and down) for incident radiation reaching the structure from vertical interfaces. However, due to multiple reflections in the structure elements, the actual number of X-ray paths is usually much higher than four. Actually, as shown in Fig. 1, a fluorescence point can be reached by the incoming radiation after $0,1,2$, etc., reflections at the horizontal interfaces of the structure elements.

If structure elements differ in height then the number of ray path directions that should be considered grows but can be limited to a still tractable number.

\subsection{Roughness model}

The effects of the surface roughness can significantly change the GIXRF signal. ${ }^{4-11}$ Thus, it is of paramount importance to introduce a roughness model into the ray tracing method.

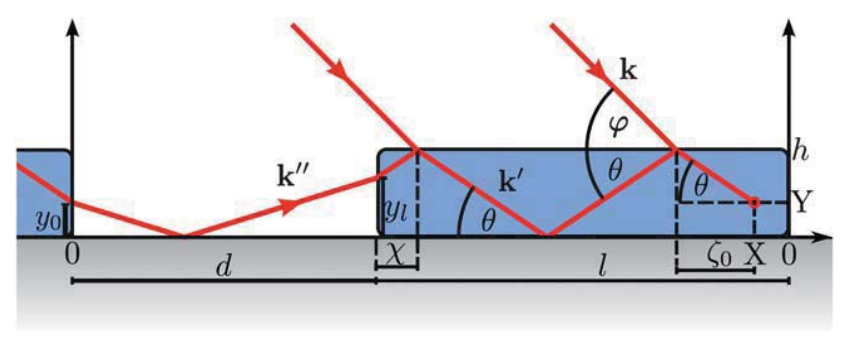

Fig. 1 Graphical representation of a ray path in a rectangular island structure. The symbols are explained in the text. 
In this work a very simple roughness model is used that neglects the roughness of the support. Keeping in mind the above stated assumption about the structures' interfaces that should be either horizontal or vertical, the top interface roughness can be introduced as a simple height variation $h(x)$ of the structure with a standard deviation $\sigma_{h}$ corresponding to the rms of the roughness. However, it should be noted that in this simplified roughness model the effects of the radiation scattering at the interface are not considered.

\subsection{Reverse ray tracing algorithm}

Let us consider an absorption point of coordinates $[X, Y]$ located in an island of length $l$, variable height $h(x)$ and distant by $d$ from the previous neighboring island.

The incident radiation can be characterized by the following ray vector $\boldsymbol{k}$ :

$$
\boldsymbol{k}=\left(k_{x},-k_{y}\right)=k(\cos \varphi,-\sin \varphi)
$$

where $k$ is the radiation wave number and $\varphi$ is the angle of incidence. The wave vector $\boldsymbol{k}^{\prime}$ of the radiation refracted into the structure can be described as follows - if the radiation reaches the vertical interface:

$$
\boldsymbol{k}_{0}^{\prime}=\left(\sqrt{(n k)^{2}-k_{y}^{2}},-k_{y}\right)
$$

or, if the radiation reaches the horizontal interface:

$$
\boldsymbol{k}_{1}^{\prime}=\left(k_{x},-\sqrt{(n k)^{2}-k_{x}^{2}}\right)
$$

where $n$ stands for the refraction index for X-rays of the structure material.

If the height of the structure elements varies then the wave vectors resulting from the following recursive equations are taken into consideration (see Fig. 2):

$$
\begin{aligned}
& \boldsymbol{k}_{0}^{\prime}=\left(\sqrt{(n k)^{2}-k_{y}^{2}},-k_{y}\right), \\
& \boldsymbol{k}_{i+1}^{\prime}=\left(\sqrt{k^{2}-k_{i y}^{\prime 2}},-\sqrt{(n k)^{2}-k^{2}+k_{i y}^{\prime}{ }^{2}}\right), \\
& \boldsymbol{k}_{i-1}^{\prime}=\left(\sqrt{(n k)^{2}-k^{2}+k_{i x}^{\prime}},-\sqrt{k^{2}-k_{i x}^{\prime}}{ }^{2}\right) .
\end{aligned}
$$

It should be noted that the $\boldsymbol{k}_{0}^{\prime}$ and $\boldsymbol{k}_{1}^{\prime}$ values given by eqn (2) and (3) can be retrieved from the first and second equations $(i=0)$ of eqn (4). The number of wave vectors $\boldsymbol{k}_{i}$ to consider in simulation should be limited to a reasonable number.

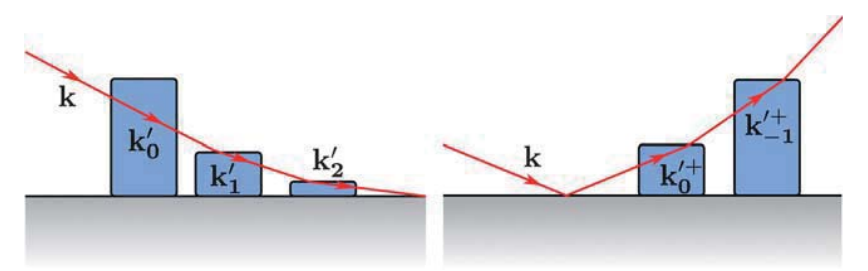

Fig. 2 Various wave vectors as described in the text.
For a given wave vector $\boldsymbol{k}^{\prime}$ of the radiation refracted into the structure the ray paths with the following final wave vectors have to be considered: $\boldsymbol{k}^{\prime}=\left(k_{x}^{\prime}, k_{y}^{\prime}\right)$ and $\boldsymbol{k}^{\prime+}=\left(k_{x}^{\prime},-k_{y}^{\prime}\right)$.

The angle of incidence of the refracted radiation is given by:

$$
\theta=\arctan \left|\frac{\operatorname{Re}\left(k_{y}^{\prime}\right)}{\operatorname{Re}\left(k_{x}^{\prime}\right)}\right|,
$$

where $\operatorname{Re}(z)=\frac{1}{2}(z+\bar{z})$ stands for the real part of the complex number $z$. The lateral distance between the absorption point and the last reflection on the top interface of the island can be written as:

$$
\zeta_{0}=\left\{\begin{array}{lc}
\left(h_{1}-Y\right) \cot \theta & \text { for } \boldsymbol{k}^{\prime} \\
\left(h_{1}+Y\right) \cot \theta & \text { for } \boldsymbol{k}^{\prime+}
\end{array}\right.
$$

whereas for the ray paths where a higher number of reflections occur the distance between two consecutive reflections on the top interface of the island is given by:

$$
\zeta_{i}=\left(h_{i-1}+h_{i}\right) \cot \theta,
$$

where $h_{i}$ is the island's height at the $i^{\text {th }}$ top interface reflection. The maximal number $N$ of top interface reflections in the island is:

$$
N=\min \left\{\tilde{N} \in \mathbb{N}_{0}: \sum_{i=0}^{\tilde{N}} \zeta_{i}>l-X\right\} .
$$

A longer ray path, entering the island by a vertical interface, covers an additional distance $\chi$ :

$$
\chi=l-X-\sum_{i=0}^{N} \zeta_{i},
$$

and enters the island at the height $y_{l}$ :

$$
y_{l}=\left|h_{N}-\chi \tan \theta\right| \text {. }
$$

At the vacuum side the ray path has the wave vector $\boldsymbol{k}^{\prime \prime}$ of the following form:

$$
\boldsymbol{k}^{\prime \prime}=\left(\sqrt{k^{2}-k_{y}^{\prime 2}}, \operatorname{sgn}\left(\chi \tan \theta-h_{N}\right) k_{y}^{\prime}\right)
$$

and the ray path crosses the position of the vertical interface of the previous neighbouring island at the height of:

$$
y_{0}=\left|y_{l}+d \frac{\operatorname{Re}\left(k_{y}^{\prime \prime}\right)}{\operatorname{Re}\left(k_{x}^{\prime \prime}\right)}\right| .
$$

If the following condition is fulfilled:

$$
y_{0}<h
$$

the ray tracing procedure can be continued for coordinates relative to the previous neighbouring island starting at the point $\left[0, y_{0}\right]$.

If the condition (13) is not fulfilled and $k_{y}^{\prime \prime}>0$, then the ray tracing procedure can be continued for the ray vector $\boldsymbol{k}_{i+1}^{\prime+}$ and 
coordinates relative to the previous neighbouring island starting at the point $\left[\frac{\operatorname{Re}\left(k_{x}^{\prime \prime}\right)}{\operatorname{Re}\left(k_{y}^{\prime \prime}\right)}\left(h-y_{0}\right), h\right]$ provided that:

$$
-l<\frac{\operatorname{Re}\left(k_{x}^{\prime \prime}\right)}{\operatorname{Re}\left(k_{y}^{\prime \prime}\right)}\left(h-y_{0}\right) .
$$

For the ray vectors $\boldsymbol{k}_{i>0}^{\prime}$ also the rays refracted at the top interface should be checked for the condition (13) with $y_{0}$ defined as follows:

$$
y_{0}=h_{j}+\left(d+l-x_{j}\right) \frac{\operatorname{Re}\left(k_{y}^{*}\right)}{\operatorname{Re}\left(k_{x}^{*}\right)},
$$

where $\left[h_{j}, x_{j}\right]$ are the coordinates of the refraction point and $\boldsymbol{k}^{*}$ is the wave vector at the vacuum side of the horizontal interface:

$$
\boldsymbol{k}^{*}=\left(k_{x}^{\prime},-\sqrt{k^{2}-k_{x}^{\prime 2}}\right) .
$$

If the condition (13) is fulfilled the ray tracing procedure can be continued for the wave vector $\boldsymbol{k}_{i-1}$ and the coordinates relative to the previous neighbouring island starting at the point $\left[0, y_{0}\right]$.

If the condition (13) is not fulfilled and if the wave vector of the considered ray path after the first refraction has the form (2) or (3), then the resulting radiation amplitude evolution is calculated and added to the radiation intensity at the absorption point.

The amplitude evolution $E$ across the ray path follows the wave equation:

$$
E=E_{0} \prod_{j, k, l} r_{k} t_{l} \exp \left\{-i \Delta x_{j}\left(k_{j x}+k_{j y} \frac{\operatorname{Re}\left(k_{j y}\right)}{\operatorname{Re}\left(k_{j x}\right)}\right)\right\},
$$

where $E_{0}$ is the initial amplitude, $\Delta x_{j}$ is the lateral distance between consecutive top interface reflections and/or refractions on vertical interfaces $(e . g ., \zeta$ and $\chi),\left(k_{j x}, k_{j y}\right)$ is the wave vector at the distance $\Delta x_{j}$, and $r_{k}$ and $t_{l}$ stand respectively for the reflection and transmission coefficients for all reflections and transmissions encountered in the ray path.

The ray tracing procedure is stopped whenever the ray path amplitude calculated with (17) becomes smaller than a previously chosen threshold. In order to obtain information about the whole sample, the reverse ray tracing should be performed for as many absorption points as needed.

For incidence angles close to zero, the algorithm may diverge, depending on the sample. GO calculations at very shallow angles should thus be performed cautiously.

\subsection{Optical paths coherence}

For the interference effects to occur the interfering optical paths need to be coherent. This implies that the length difference between the optical paths should be within the radiation coherence length $\xi$.

In the case of GIXRF the coherence length depends on the radiation source parameters and can reach up to several microns for third generation synchrotron radiation sources. ${ }^{12}$
The resulting intensity $I$ can be calculated with the following integral:

$$
I=\int \mathrm{d} x\left|\sum_{i} E_{i} C_{\xi}\left(x-L_{i}\right)\right|^{2},
$$

where $E_{i}$ is the electric field of the ray path $i$ at the absorption point (calculated with (17)), $C_{\xi}(x)$ is the coherence correlation function characterized by the coherence length $\xi$, and $L_{i}$ is the length of the optical path $i$.

In the above equation only the amplitudes of the ray paths which have some degree of coherence, i.e., $C_{\xi}\left(L_{i}-L_{j}\right) \neq 0$, are taken into consideration in the sum. If the length difference between the optical paths is too high, i.e., $C_{\xi}\left(L_{i}-L_{j}\right)=0$, only the summing of the squared amplitudes is performed $I=$ $\sum_{i}\left|E_{i}\right|^{2}$.

\subsection{Effective flux}

In the case of granular structures a part of the incident radiation might be reflected on the substrate surface without reaching the on-surface objects. As a consequence, a part of the incoming beam intensity does not contribute to the fluorescence production of the structure of interest. Moreover, the relative intensity of this inactive beam part changes as a function of the grazing incidence angle. In order to properly calculate the angular evolution of the fluorescence intensity for a granular structure the beam flux $\Phi$ therefore has to be corrected.

The incident radiation can be divided into three parts (see Fig. 3):

(I) the radiation reaching the structure elements directly,

(II) the radiation reaching the structure after a reflection,

(III) the radiation which never reaches the structure.

The effective flux $\Phi_{\text {eff }}$ that should be considered can be given with a ratio $\sigma$ :

$$
\Phi_{\text {eff }}=\sigma \Phi=\frac{\mathrm{I}+\mathrm{II}}{\mathrm{I}+\mathrm{II}+\mathrm{III}} \Phi .
$$

The calculation of $\Phi_{\text {eff }}$ requires the knowledge of the distribution and dimensions of the on-surface objects and in most cases it cannot be obtained in a simple formula. Still, the general rule is that $\sigma$ is equal to the covering rate $R$ of the structure if the radiation is perpendicular to the surface and reaches one for very shallow angles.

As an example let us consider a 2D structure consisting of evenly distributed squares with a side length $a$, the distance

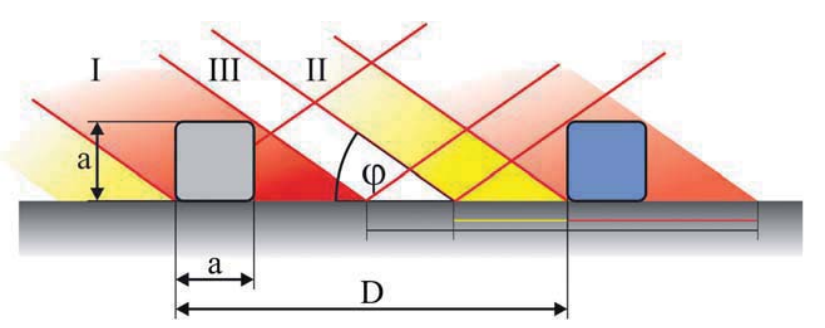

Fig. 3 Illustration of the effective flux for an on-surface structure of evenly distributed squares and an incidence angle $\varphi$ of the incoming radiation. 
between two consecutive squares being $D$ (see Fig. 3). For the radiation perpendicular to the surface $\sigma$ is equal to the covering rate of the structure, i.e., $R=a / D$. For smaller incidence angles, $\sigma$ can be derived from the following formula:

$$
\sigma(\varphi)= \begin{cases}R(1+2 \cot \varphi) & \text { if } \varphi>\arctan \frac{D-a}{2 a} \\ 1 & \text { else. }\end{cases}
$$

For a well characterized structure $\sigma$ is calculated using the structure parameters obtained from SEM or AFM images.

It can be noted here that the effective flux correction might be also useful for ultra-shallow buried implant samples as those discussed in ref. 13.

\section{Materials and methods}

The experimental GIXRF data were obtained in a measurement campaign in the laboratory of the Physikalisch-Technische Bundesanstalt (PTB) at the electron storage ring BESSY II. ${ }^{14}$ The samples were mounted in the PTB's X-ray spectrometry chamber. This ultra-high vacuum chamber allows for experiments under conventional XRF conditions as well as in the GIXRF geometry with incident angles up to $5^{\circ}$. In Fig. 4 the layout of the experimental arrangement is shown. Monochromatic synchrotron radiation with a well-known flux and intensity distribution in the focal plane is used to excite the sample under different incident angles. The reproducibility of the incident angle lies within $0.005^{\circ}$. The projection of the beam onto the sample surface, the so-called footprint of the beam, can be calculated and therefore for all incident angles the effective solid angle of detection is known, thanks to the calibrated geometry of the setup. The fluorescence radiation emitted by the sample material is detected with a silicon drift detector (SDD) positioned close to the sample surface.

Two types of nanoscaled sample systems were investigated: monodisperse $\mathrm{NaCl}$ nanocrystals deposited from aerosol phase and artificial chromium pads' structures..$^{15,16}$

The $\mathrm{NaCl}$ nanoparticles were first sorted according to their size with a scanning mobility particle sizer and then deposited on a Si surface by means of an electrostatic aerosol sampling method at the German Federal Institute for Materials Research and Testing (BAM). SEM pictures revealed that the $\mathrm{NaCl}$
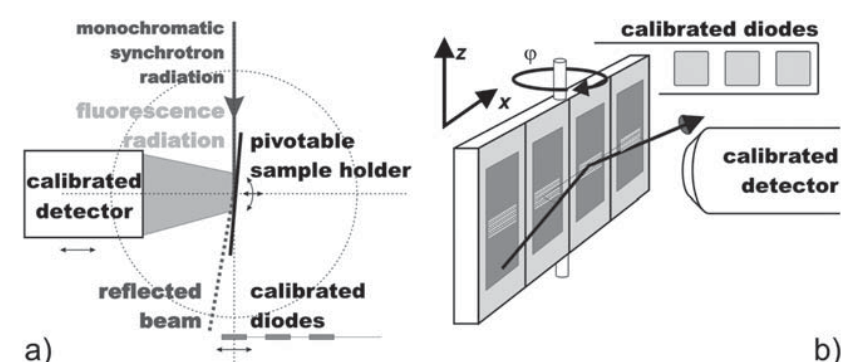

b)
Fig. 4 Sketch of the experimental setup. (a) Top view. The energy-dispersive silicon drift detector is aligned perpendicularly to the incident beam, thus minimizing the detection of scattered radiation. (b) View from the upstream side. One can see the rows of structures that were carefully aligned to be parallel to the incoming beam direction. nanoparticles (see the left panel of Fig. 5) form monodisperse single crystals of similar size with an average particle dimension of $50 \mathrm{~nm}$ and a density of $6 \times 10^{6}$ particles per $\mathrm{mm}^{2}$. The measurements were performed at the plane grating monochromator beamline (PGM) ${ }^{17}$ for undulator radiation which provides photons in the range of $78 \mathrm{eV}$ to $1870 \mathrm{eV}$.

The second sample was produced by a lift-off procedure. A pattern of 2250 circular holes with a diameter of $2.7 \mu \mathrm{m}$, arranged in 7 lines along the wafer surface, was imprinted into a photoresist by electron beam lithography. The whole surface was covered with a $50 \mathrm{~nm}$ thick layer of chromium. After removal of the photoresist, $50 \mathrm{~nm}$ high chromium cylinders remained on the surface in the pattern defined by the lithography (see the right panel of Fig. 5). The minimal distance between two of those cylinders was $50 \mu \mathrm{m}$. Thus, only about $0.2 \%$ of the surface was covered with the Cr pads. The experiment was set up at the four-crystal monochromator beamline $(\mathrm{FCM})^{18}$ that provides bending magnet radiation in the X-ray regime between $1.75 \mathrm{keV}$ and $10.5 \mathrm{keV}$. Thus, the $\mathrm{Cr}-\mathrm{K}$ shell was accessible.

The recorded X-ray fluorescence spectra were deconvoluted with known detector response functions. ${ }^{19}$ Thus, with the also known effective solid angle of detection, the actual emitted fluorescence intensity could be calculated from the measured count rates for each incident angle. For nanoscaled sample systems, where self-absorption effects are negligible, the emitted fluorescence intensity is proportional to the effective excitation intensity which can be calculated with either the XSW or the GO approach.

To get reliable data with a high enough statistics, the $\mathrm{K} \alpha \mathrm{X}$ ray lines of $\mathrm{Na}$ and $\mathrm{Cr}$ were measured using an acquisition time of $45 \mathrm{~s}$ for each incident angle.

\section{Experimental results and calculations}

\subsection{NaCl nanocrystals}

In order to obtain the spatial distribution of the NaCl nanoparticles and their size distribution, SEM images of the sample were evaluated. Although these SEM images do not give explicit information on the structures height, the height of the particles was assumed to be proportional to the square root of the area occupied by the individual particles. Such an assumption is justified assuming a uniform growth of the $\mathrm{NaCl}$ nanocrystals in all three dimensions - length, width and height.

The experimental GIXRF angular profile of the $\mathrm{NaCl}$ sample was determined by measuring the variation of the $\mathrm{Na} \mathrm{K} \alpha$ fluorescence intensity as a function of the incidence angle of the incoming X-ray beam. The results are depicted in Fig. 6 where they are compared to the theoretical profiles calculated with the XSW and GO models. Note that the XSW curve was obtained from the linear combination of weighted XSW simulations performed using particle sizes and corresponding weights according to the distribution deduced from the SEM images and that the GO curve was corrected to account for the above mentioned effective beam flux.

From Fig. 6 one can first see that the theoretical simulations which have a similar form do reproduce both the overall shape 

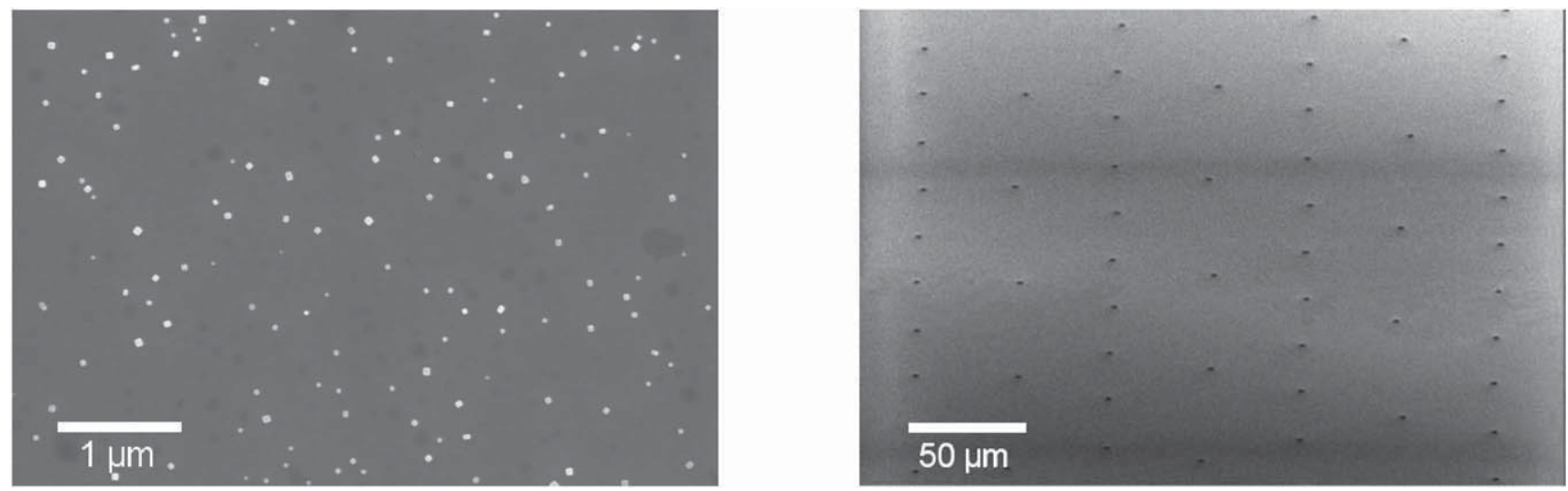

Fig. 5 SEM images of the investigated samples; left: $\mathrm{NaCl}$ nanostructures; right: $\mathrm{Cr}$ pads. ${ }^{15}$

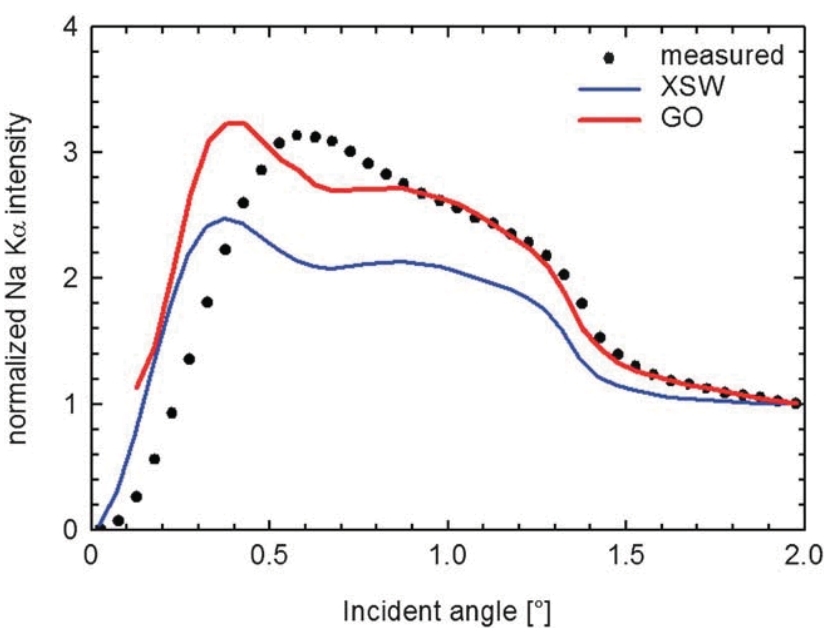

Fig. $6 \mathrm{Na} \mathrm{K \alpha}$ GIXRF profile of $\mathrm{NaCl}$ nanostructures measured with an incident photon beam of $1260 \mathrm{eV}$. Black dots: measured data; blue line: XSW simulation; red line: GO reverse ray tracing simulation corrected for the effective flux. Experimental and theoretical data were normalized to 1 at the largest incidence angle.

of the measured profile consisting of a fast growth of the intensity at shallow angles followed by a slightly decreasing intensity region between $0.5^{\circ}$ and $1.3^{\circ}$ and a rapid intensity decrease around the critical angle at about $1.4^{\circ}$. One can also see that the GO predictions are in general closer to the experimental data than the XSW ones, a very nice agreement being even observed between the measured intensity and the GO curve above about $0.9^{\circ}$.

In contrast to that, important deviations can be seen between the measured profile and the two theoretical curves in the fast increase region and around the maximum, indicating that for this sample both models are not able to provide the correct fluorescence intensity at shallow angles. The two-bump structure predicted by both simulations is also smeared out and hardly visible in the measured profile.

In the reference-free quantification method used in our experiment, the main contributions to the total experimental uncertainty are the uncertainties on the atomic fundamental parameters (about 10\%) and the ones concerning the effective solid angle of detection and detector efficiency (about 3\%), the statistical errors of the experimental points being negligibly small. As a consequence, the discrepancies discussed above cannot be explained by the experimental uncertainties. We are rather inclined to believe that these discrepancies are mainly due to the following four reasons: (1) the roughness of the substrate surface that was not taken into consideration in the simulations and could additionally disturb the X-ray reflection at shallow angles; (2) the nonuniform $\mathrm{NaCl}$ crystal growth that would result in a different height distribution; (3) the difficulties encountered at small incidence angles with the normalization of the fluorescence intensity; and (4) the FWHM of the particle size distribution which is about $10 \%$ even under optimal conditions. This size scatter smears out to a certain extent the GIXRF angular resolution and probably also contributes to the observed deviations between the XSW and GO simulations on the one hand and the experimental data on the other hand.

\subsection{Cr pads}

The Cr pads structures were designed in such a way that the normalization for the geometrical effects was minimized. ${ }^{15}$ Thus, the correction for the effective flux was not necessary. Because the chromium pads structures were prepared with the lift-off technique all morphology parameters could be taken directly from the sample specification. Some SEM images were recorded which validated the sample geometry (see Fig. 5). The height of the $\mathrm{Cr}$ structures was verified by SEM-EDX measurements. ${ }^{20}$

The measured angular profile of the $\mathrm{Cr}$ pads structure is depicted in Fig. 7 together with the results of the XSW and GO simulations. As shown, the XSW calculations reproduce only roughly the measured GIXRF spectrum. The XSW curve exhibits a fast intensity growth at shallow incident angles and pronounced interference fringes which are not observed in the experimental profile.

The GO simulation, even if not taking into account the surface roughness, fits the data far better (dashed red line in Fig. 7). The experimental intensity growth at shallow angles is well reproduced because the shadowing effect of consecutive 


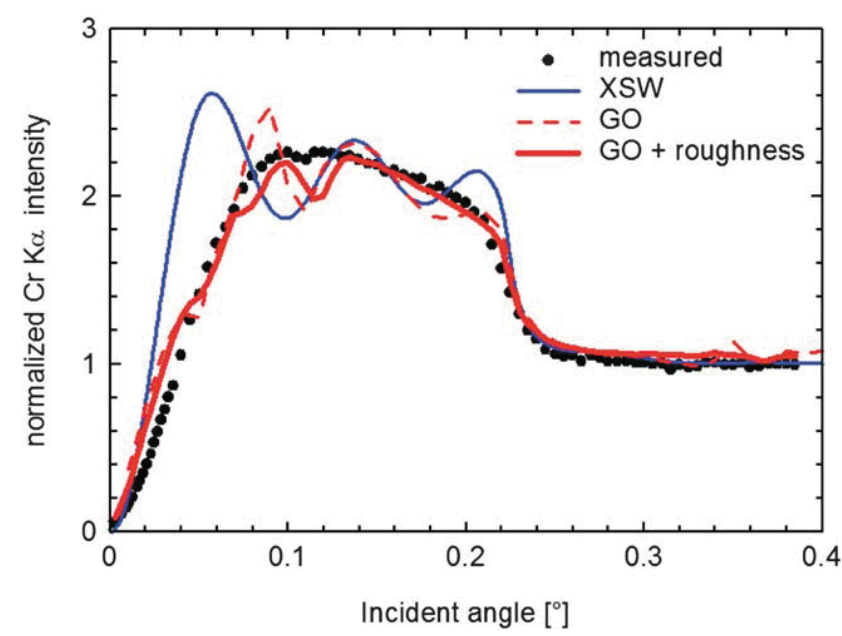

Fig. $7 \mathrm{Cr} \mathrm{K} \alpha$ GIXRF profile of the $50 \mathrm{~nm}$ high chromium pads measured with an incident photon beam of $8040 \mathrm{eV}$. Black dots: measured data; blue line: XSW simulation of a single pad; red dashed line: $\mathrm{GO}$ reversed ray tracing simulation with no surface roughness; thick red line: GO reversed ray tracing simulation including the effect of the surface roughness of $5 \mathrm{~nm} \mathrm{rms}$ and the influence of the shadowing of consecutive pads. Experimental and theoretical data were normalized to 1 at the largest incidence angle.

pads is taken into account in the GO method but not in the XSW one. Also the intensity decrease around the critical angle is very close to the measured data, thanks to the consideration of the intensity attenuation effects in the GO approach.

Furthermore, as shown the interference fringes smear out and the experimental data are still better reproduced if an additional pad's surface roughness of $5 \mathrm{~nm}$, which is a realistic value, is introduced in the GO calculations (solid red line in Fig. 7). In contrast to the currently used models (XSW) an excellent agreement between the GO predictions including the effect of the sample surface roughness and the experimental data is indeed observed.

\section{Conclusions}

The GO approach leads to very good results when compared to absolute measurements recorded at higher photon energies and to the well-established XSW model. GO can handle the absorption and shadowing effects occurring on the nanostructured surfaces which are very difficult to simulate with XSW. Also it is relatively easy to incorporate specific sample geometries into the GO calculations.

On the other hand the XSW method needs no input about the sample geometry and with available software, such as IMD, ${ }^{21}$ the simulations can be calculated very quickly. Thus, for standard situations where the surface morphology effects are not pronounced the GO methodology can hardly compete with XSW.

Nevertheless, for the case of particulate media and buried implants (depth profiling) the correction for the effective flux, that also requires an initial input about the sample geometry, cannot be neglected.

GO is certainly a promising approach for samples with a known surface morphology where the a priori knowledge of the structure can be verified. This approach might be also helpful to understand uncommon GIXRF profiles of rough layers and grainy structures. In the nearest future the presented methodology will be extended to a wider range of sample systems.

We believe that systematic GO simulations can significantly contribute to the development of more precise TXRF quantification models for particulate media. Such a new quantification method would be especially useful for densely packed nanodevices or nanoparticles sampled directly from the aerosol phase and from dried liquid droplets. GO simulations may also substantially contribute to the further development of the XSW approximation scheme for high nanoparticle or structure depositions.

\section{Acknowledgements}

The project was partly supported by the Swiss National Science Foundation. Some of the authors (F.R. and B.B.) thank the Investitionsbank Berlin for financial support of the ProFIT/ EFRE project 'nanoparticle X-ray analytics' as well as Harald Bresch and Stefan Seeger, German Federal Institute for Materials Research and Testing (BAM), for providing the $\mathrm{NaCl}$ nanoparticles.

\section{References}

1 A. von Bohlen, Spectrochim. Acta, Part B, 2009, 64, 821-832.

2 A. von Bohlen, M. Krämer, C. Sternemann and M. Paulus, J. Anal. At. Spectrom., 2009, 24, 792.

3 R. Klockenkämper, Total-Reflection X-Ray Fluorescence Analysis, Wiley-Interscience, 1996.

4 L. Nevot, B. Pardo and J. Corno, Rev. Phys. Appl., 1988, 23, 1675-1686.

5 H. Schwenke, R. Gutschke, J. Knoth and M. Kock, Appl. Phys. A: Solids Surf., 1992, 54, 460-465.

6 K. Tsuji, T. Yamada, T. Utaka and K. Hirokawa, J. Appl. Phys., 1995, 78, 969-973.

7 D. K. G. de Boer, Phys. Rev. B: Condens. Matter, 1994, 49, 5817-5820.

8 W. Wu and W. Wallace, J. Vac. Sci. Technol., B, 1998, 16, 1958.

9 K. Tsuji, S. Sato and K. Hirokawa, Thin Solid Films, 1996, 274, 18-22.

10 D. K. G. de Boer, Phys. Rev. B: Condens. Matter, 1995, 51, 5297-5305.

11 D. K. G. de Boer, Phys. Rev. B: Condens. Matter, 1996, 53, 6048-6064.

12 I. A. Vartanyants and A. Singer, New J. Phys., 2010, 12, 035004.

13 P. Hönicke, Y. Kayser, B. Beckhoff, M. Müller, J.-Cl. Dousse, J. Hoszowska and S. H. Nowak, J. Anal. At. Spectrom., 2012, 27, 1432-1438.

14 B. Beckhoff, A. Gottwald, R. Klein, M. Krumrey, R. Müller, M. Richter, F. Scholze, R. Thornagel and G. Ulm, Phys. Status Solidi B, 2009, 246, 1415-1434.

15 F. Reinhardt, J. Osán, S. Török, A. E. Pap, M. Kolbe and B. Beckhoff, J. Anal. At. Spectrom., 2012, 27, 248-255. 
16 J. Osán, F. Reinhardt, B. Beckhoff, A. E. Pap and S. Török, ECS Trans., 2009, 25, 441-451.

17 F. Senf, U. Flechsig, F. Eggenstein, W. Gudat, R. Klein, H. Rabus and G. Ulm, J. Synchrotron Radiat., 1998, 5, 780-782.
18 M. Krumrey, J. Synchrotron Radiat., 1998, 5, 6-9.

19 F. Scholze and M. Procop, X-Ray Spectrom., 2001, 30, 69-76.

20 C.-U. Ro, J. Osán, I. Szalóki, J. de Hoog, A. Worobiec and

R. V. Grieken, Anal. Chem., 2003, 75, 851-859.

21 D. Windt, Comput. Phys., 1998, 12, 360. 